\title{
Short communication: Changes in fluorescence intensity induced by soybean soluble polysaccharide-milk protein interactions during acidification
}

\author{
Y. H. Li, ${ }^{*}$ W. J. Wang, $†$ X. J. Xu, ${ }^{*}$ Y. C. Meng, ${ }^{* 1}$ L. W. Zhang,‡ J. Chen, ${ }^{*}$ and R. Qiu* \\ ${ }^{*}$ College of Food Science and Biotechnology Engineering, Zhejiang Gongshang University, Hangzhou 310018, China \\ †Beingmate Baby \& Child Food Co., Ltd., Hangzhou 311106, China \\ $\ddagger$ School of Food Science and Engineering, Harbin Institute of Technology, Harbin 150090, China
}

\section{ABSTRACT}

Interactions between stabilizer and milk protein are believed to influence the stabilizing behavior of the milk system. We investigated changes in fluorescence intensity induced by interactions of soybean soluble polysaccharide (SSPS) and milk protein (Mp) during acidification. The fluorescence intensity $\left(\mathrm{I}_{\mathrm{f}}\right)$ of $\mathrm{Mp}$ increased as $\mathrm{pH}$ decreased from 6.8 to 5.2. Compared with Mp alone, $\mathrm{I}_{\mathrm{f}}$ of SSPS-Mp mixtures increased as the $\mathrm{pH}$ decreased from 6.8 to 5.2. We found that the $\mathrm{I}_{\mathrm{f}}$ of the SSPS-Mp mixture decreased in a $\mathrm{pH}$ range from 5.2 to 3.6 , which indicated a change in the polarity microenvironment around the Trp residues. We also found that the maximum emission wavelength $\left(\lambda_{\max }\right)$ shifted from 337 to $330 \mathrm{~nm}$ as $\mathrm{pH}$ decreased from 6.8 to 3.6, in further support of SSPS interacting with the polar portion of Mp during acidification. Furthermore, an excited monomeric molecule (pyrene exciplex) was found as a ground-state pyrene formed and a broad band was shown at about $450 \mathrm{~nm}$. The intensity ratio of the first peak to the third peak $\left(\mathrm{I}_{1}: \mathrm{I}_{3}\right)$ of $\mathrm{Mp}$ increased slightly, and the ratio of intensity of pyrene exciplex to monomer $\left(\mathrm{I}_{\mathrm{e}}: \mathrm{I}_{\mathrm{m}}\right)$ decreased because pyrene molecules were located in a less hydrophobic microenvironment during acidification. However, the ratio of $\mathrm{I}_{1}: \mathrm{I}_{3}$ decreased clearly at $\mathrm{pH}$ below 5.6 and the ratio of $\mathrm{I}_{\mathrm{e}}: \mathrm{I}_{\mathrm{m}}$ showed the opposite trend in the SSPS-Mp mixture. Changes in intrinsic and exogenous fluorescence intensity confirmed that interactions of SSPS and Mp could change the polarity of the microenvironment and that SSPS probably interacted with the polar portion of Mp. These results could give insight into the behavior of stabilizers in acid milk products.

Key words: soybean soluble polysaccharide, milk protein, fluorescence intensity, acidification

Received June 16, 2015.

Accepted August 26, 2015.

${ }^{1}$ Corresponding author: mengyuecheng126@sina.com

\section{Short Communication}

Caseins are the main proteins in milk and they play a major role in imparting structure to dairy products. In general, caseins are solubilized in the form of casein micelles under neutral $\mathrm{pH}$ conditions and they precipitate or aggregate under acidic $\mathrm{pH}$ conditions. The $\mathrm{pH}$ of acidified milk drinks ranges from 3.4 to 4.6 , and because of the instability of caseins in this $\mathrm{pH}$ range, a stabilizer needs to be added to prevent protein aggregation and achieve optimal mouthfeel (Nakamura et al., 2006; Corredig et al., 2011).

Protein-polysaccharide interactions are important in the formulation of acidified milk drinks. Soybean soluble polysaccharide (SSPS) extracted from soybean cotyledons is often used to stabilize acidic beverages, which could improve the stability and texture of acidified milk drinks under low $\mathrm{pH}$ (acidic) conditions (Pereyra et al., 1997). Soybean soluble polysaccharide is negatively charged and fully adsorbs to the surface of positively charged proteins in acidic beverages. The steric repulsive force of SSPS adsorbed on the surface of casein explains stability at low $\mathrm{pH}$, and the electrostatic repulsive force of polysaccharide chains contributes to the ability of SSPS to disperse milk proteins (Nakamura et al., 2003; Nobuhara et al., 2014).

The fluorescence in milk allows monitoring of structural modifications of proteins and their physicochemical environment during the coagulation process. Herbert et al. (1999) showed that a fluorescence method allowed detection of structural changes in milk during coagulation and discrimination of different dynamics of the coagulation system. In milk, most of the intrinsic fluorescence intensity can be attributed to the caseins present in the casein micelles $\left(\alpha_{\mathrm{S1}^{-}}, \alpha_{\mathrm{S}^{2}}, \beta-\right.$, and $\kappa$-caseins) and whey proteins ( $\alpha$-LA, $\beta-\mathrm{LG}, \mathrm{BSA}$; Fox, 1989; Tayeh et al., 2009; Rahimi and Corredig, 2012). The fluorescence of protein usually comes from the residues of Phe, Tyr, and Trp; Trp fluorescence is widely used to study interactions between protein and other molecules because of the high sensitivity to the polarity of its environment (Liu and Guo, 2008a,b). Be- 
cause $\alpha_{S}$-casein and BSA have 2 Trp residues, $\beta$-casein and $\kappa$-casein have 1 Trp residue, and $\alpha$-LA has $4 \operatorname{Trp}$ residues (Kronman et al., 1972; Fox, 1989; Vanhooren et al., 2006; Tayeh et al., 2009; Rahimi and Corredig, 2012), structural alterations of the proteins could be detected by Trp fluorescence parameters. It could provide important information about casein micelles as well as the exogenous probe (Chakraborty and Basak, 2007).

In this study, we measured the fluorescence intensity of milk protein (Mp) alone and SSPS-Mp complex as a function of $\mathrm{pH}$ value. We aimed to study the interaction of Mp and SSPS during acidification and to evaluate the kinetics of coagulation and structural aspects of the stabilizing layer. Our results could have important theoretical and practical implications in the stabilization of acid milk products.

Skim milk powder was obtained from Fonterra Cooperative Group (Wellington, New Zealand). The SSPS was donated by Fuji Oil Co. Ltd. (Osaka, Japan) and was prepared from the residue of protein extraction, as previously described (Nakamura et al., 2001, 2006). Pyrene was purchased from Fluka Company (Buchs, Switzerland), and citric acid was obtained from Zhanwang Chemical Co. Ltd. (Wuxi, China).

The reconstituted milk with a content of $2 \%$ (wt/ vol) protein was prepared by mixing skim milk powder with milli-Q water (Millipore Corporation, Billerica, MA). Meanwhile, SSPS was prepared in deionized water, stirred at $70^{\circ} \mathrm{C}$ for $30 \mathrm{~min}$, and stored at room temperature to allow complete hydration. The final concentration in mixtures was calculated to obtain samples with $1 \%$ (wt/vol) proteins and $0.5 \%$ (wt/vol) SSPS; the mixtures were made from the reconstituted milk and SSPS hydrates.

Determination of Trp fluorescence was performed by using an F-7000 type spectrometer (Hitachi, Tokyo, Japan). The SSPS-Mp mixtures prepared with different $\mathrm{pH}$ values were diluted 10 times before determination of Trp fluorescence. The excitation and emission slits were fixed at 3.0 and $1.5 \mathrm{~nm}$, respectively. The excitation wavelength was set at $297 \mathrm{~nm}$ and the emission spectra were collected from 290 to $400 \mathrm{~nm}$. The voltage was set at $400 \mathrm{~V}$. Pyrene was used as a neutral hydrophobic probe to determine the microenvironment polarity of Mp. The emission spectra were measured at the range of 300 to $600 \mathrm{~nm}$, and the excitation wavelength was $335 \mathrm{~nm}$.

Experimental data were analyzed using ANOVA, and data were expressed as mean values \pm standard deviations. Duncan's multiple range test was performed for post hoc multiple comparisons with the level of significance set at $P<0.05$. All statistical analyses were performed using SPSS 18.0 software (SPSS Inc., Chicago, IL).
The fluorescence spectra of SSPS-Mp mixtures under different $\mathrm{pH}$ conditions are shown in Figure 1. The fluorescence intensity $\left(\mathbf{I}_{\mathbf{f}}\right)$ of Mp without addition of SSPS increased as $\mathrm{pH}$ decreased from 6.8 to 5.2 , which can be explained by the loose structure of casein micelles released during acidification and exposure of Trp residues. Acidification caused the loss of casein micelles, which presumably originated from proteins that had dissociated from the casein supramolecules (McMahon et al., 2009). Compared with Mp alone, $\mathrm{I}_{\mathrm{f}}$ of SSPS-Mp mixtures increased significantly as the $\mathrm{pH}$ decreased from 6.8 to $5.2(P<0.05)$. This result was probably consistent with the turbidity based on the inner filter effect of casein, whereby excitation or emission of light absorption led to a reduction in fluorescence intensity (Klockenkamper, 1997). At $\mathrm{pH}<5.2$, casein in the $\mathrm{Mp}$ samples precipitated and no fluorescence experiment was conducted. Meanwhile, no fluorescence emission was found for SSPS (the blank sample without Mp). In the SSPS-Mp mixtures, values of $\mathrm{I}_{\mathrm{f}}$ decreased as $\mathrm{pH}$ decreased from 5.2 to 3.6 , which indicated variation in the polarity microenvironment around the Trp residues as well as the inner filter effect.

We found that the maximum emission wavelength $\left(\boldsymbol{\lambda}_{\max }\right)$ shifted from 337 to $330 \mathrm{~nm}$ as $\mathrm{pH}$ decreased from 6.8 to 3.6 (Figure 1B). The $\lambda_{\max }$ of Trp residues shifts to shorter wavelengths when Trp residues move to a less polar microenvironment (Liu and Guo, 2008a,b). This finding could indicate that SSPS probably interacted with the polar portion of $\mathrm{Mp}$, whereas the nonpolar portion formed a more hydrophobic domain where Trp residues were located, resulting in a continuous decrease of $\lambda_{\max }$ during acidification.

The influence of $\mathrm{pH}$ on SSPS-Mp mixtures was also detected by the pyrene probe measurement (Figure 2 ). The intensity ratio of the first peak to the third peak $\left(\mathbf{I}_{1}: \mathbf{I}_{3}\right)$ of the fluorescence spectrum of pyrene indicated the polarity of the environment. The lower value of $\mathrm{I}_{1}: \mathrm{I}_{3}$, the greater hydrophobicity the system had (Keyes-Baig et al., 2004). An excited monomeric molecule (pyrene exciplex) was found as a ground-state pyrene form, which was observed as a broad band at about $450 \mathrm{~nm}$. The ratio of intensity of pyrene exciplex to monomer $\left(\mathbf{I}_{\mathrm{e}}: \mathbf{I}_{\mathrm{m}}\right)$ was used to provide information about hydrophobic domains in the system (Sahoo et al., 2000). Because the sample of Mp alone precipitated at $\mathrm{pH}<5.2$, no fluorescence experiment was conducted. The data were obtained from $\mathrm{pH} 6.8$ to 5.2. The ratio of $\mathrm{I}_{1}: \mathrm{I}_{3}$ increased slightly (from 1.001 to 1.018 ) and the ratio of $\mathrm{I}_{\mathrm{e}}: \mathrm{I}_{\mathrm{m}}$ decreased (from 0.282 to 0.156 ) with decreasing $\mathrm{pH}$, which indicated that pyrene molecules were located in a less hydrophobic microenvironment because of the loose structure of casein micelles during acidification. 

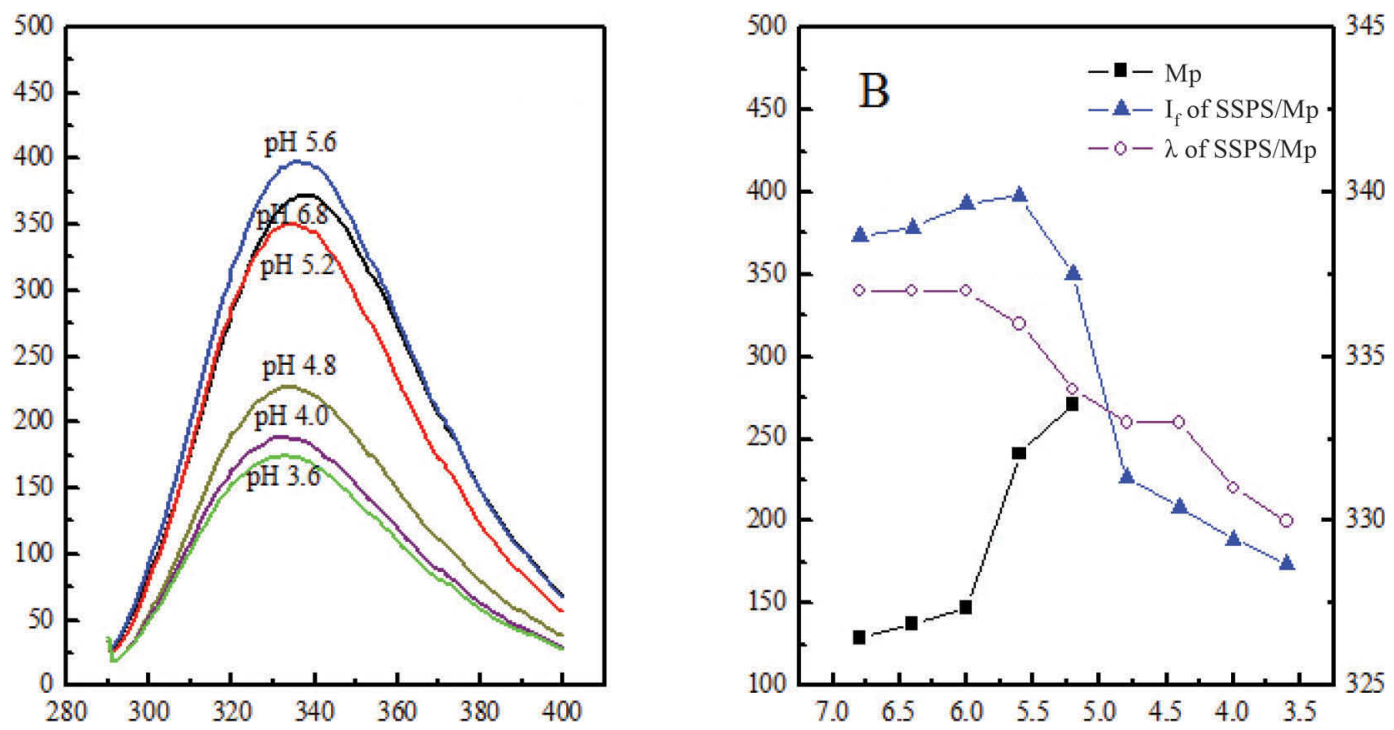

Figure 1. Fluorescence intensity changes of soybean soluble polysaccharide-milk protein (SSPS-Mp) mixtures during acidification. (A) Typical fluorescence spectra of SSPS-Mp mixtures under different $\mathrm{pH}$ conditions; (B) changes in fluorescence intensity ( $\mathrm{I}_{\mathrm{f}}$; $\mathbf{\Delta}$ ) and maximum emission wavelength $(\lambda ; \bigcirc)$ during acidification. Color version available online.
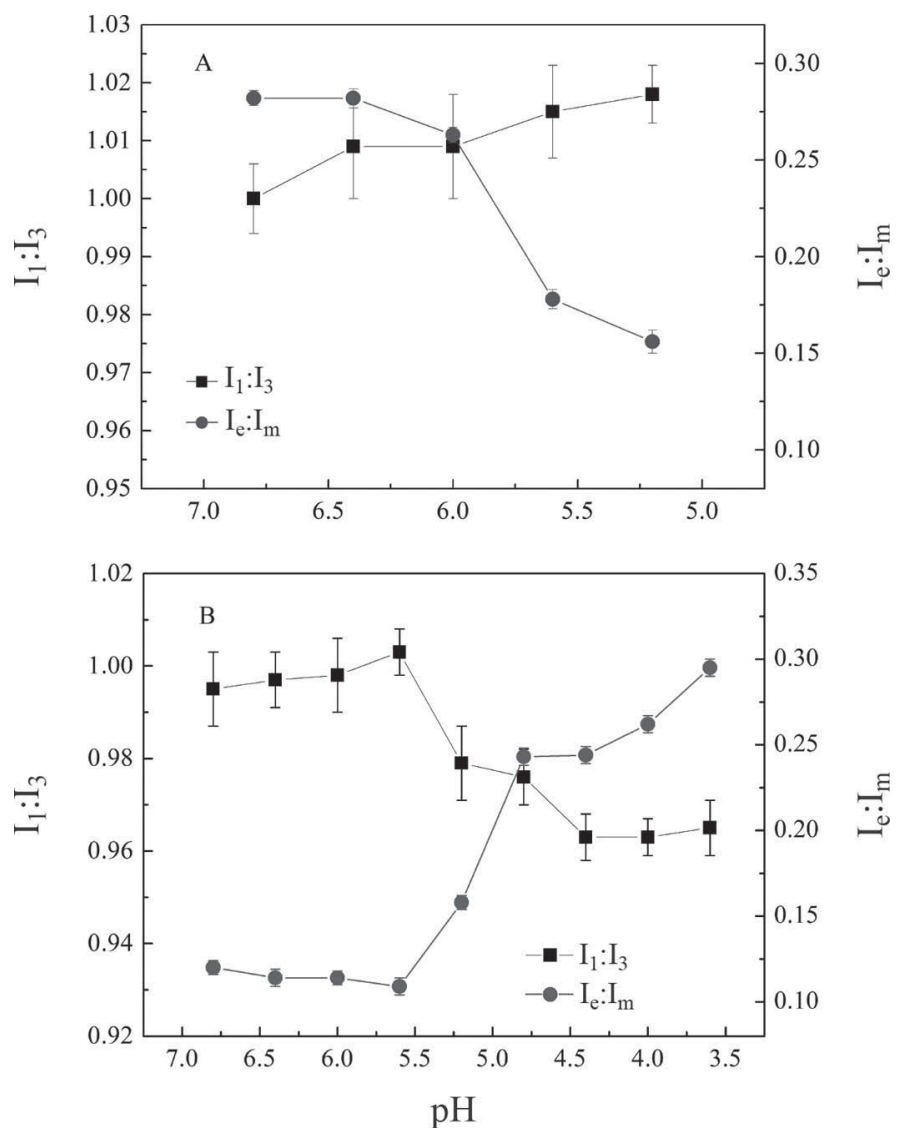

Figure 2. Changes in the intensity ratio of the first peak to the third peak $\left(\mathrm{I}_{1}: \mathrm{I}_{3}\right)$ and the ratio of pyrene exciplex intensity to monomer intensity $\left(\mathrm{I}_{\mathrm{e}}: \mathrm{I}_{\mathrm{m}}\right)$ of pyrene-labeled system during acidification. (A) Milk protein (Mp) alone; (B) soybean soluble polysaccharide-milk protein (SSPS-Mp) mixture. Means \pm SD are shown.
Figure $2 \mathrm{~B}$ shows the results of $\mathrm{I}_{1}: \mathrm{I}_{3}$ and $\mathrm{I}_{\mathrm{e}}: \mathrm{I}_{\mathrm{m}}$ in the SSPS-Mp mixture. The ratio of $\mathrm{I}_{1}: \mathrm{I}_{3}$ decreased (from 1.003 to 0.965$)$ at $\mathrm{pH}$ below 5.6, whereas the ratio of $\mathrm{I}_{\mathrm{e}}: \mathrm{I}_{\mathrm{m}}$ showed the opposite trend. Because of the interaction between Mp and SSPS during acidification, a more hydrophobic domain of MP formed and pyrene molecules were embedded in the deeper hydrophobic domain. The nonpolar portion formed more hydrophobic domain, which led to a lower ratio of $\mathrm{I}_{1}: \mathrm{I}_{3}$. Meanwhile, pyrene molecules were located in the same hydrophobic domain, which promoted the formation of exciplex and the high ratio of $\mathrm{I}_{\mathrm{e}}: \mathrm{I}_{\mathrm{m}}$ (Chandar et al., 1988). This result was consistent with the intrinsic fluorescence of Trp, which confirmed that interactions of SSPS and Mp could change the polarity of the microenvironment.

In conclusion, we demonstrated that interactions between SSPS and Mp changed the microenvironment polarity around Trp residues. We found that $\lambda_{\max }$ shifted from 337 to $330 \mathrm{~nm}$ as $\mathrm{pH}$ decreased during acidification. For SSPS-Mp mixtures, the ratios of $\mathrm{I}_{1}: \mathrm{I}_{3}$ and $\mathrm{I}_{\mathrm{e}}: \mathrm{I}_{\mathrm{m}}$ changed dramatically at $\mathrm{pH}$ below 5.6. Changes in intrinsic and exogenous fluorescence intensity confirmed that interactions between SSPS and Mp could change the polarity of microenvironment. Furthermore, SSPS probably interacted with the polar portion of $\mathrm{Mp}$.

\section{ACKNOWLEDGMENTS}

The authors acknowledge financial support from Food Science and Engineering-the most important discipline of Zhejiang Province (JYTsp20141091, Zhejiang Prov- 
ince, PR China), the Natural Science Foundation of Zhejiang Province (LY15C200003, Zhejiang Province, PR China), and the National Natural Science Foundation of the People's Republic of China (project number 31401557, PR China).

\section{REFERENCES}

Chakraborty, A., and S. Basak. 2007. pH-induced structural transitions of caseins. J. Photochem. Photobiol. B 87:191-199.

Chandar, P., P. Somasundaran, and N. Turro. 1988. Fluorescence probe investigation of anionic polymer-cationic surfactant interactions. Macromolecules 21:950-953.

Corredig, M., N. Sharafbafi, and E. Kristo. 2011. Polysaccharide-protein interactions in dairy matrices, control and design of structures. Food Hydrocoll. 25:1833-1841.

Fox, P. F. 1989. The milk protein system. Pages 1-54 in Developments in Dairy Chemistry-4. Functional Milk Proteins. P. F. Fox, ed. Elsevier Appl. Sci., New York, NY.

Herbert, S., A. Riaublanc, B. Bouchet, D. J. Gallant, and E. Dufour. 1999. Fluorescence spectroscopy investigation of acidor rennetinduced coagulation of milk. J. Dairy Sci. 82:2056-2062.

Keyes-Baig, C., J. Duhamel, S. Y. Fung, J. Bezaire, and P. Chen 2004. Self-assembling peptide as a potential carrier of hydrophobic compounds. J. Am. Chem. Soc. 126:7522-7532.

Klockenkamper, R. 1997. Total-Reflection X-Ray Fluorescence Analysis. Wiley, New York.

Kronman, M. J., W. B. Hoffman, J. Jeroszko, and G. W. Sage. 1972. Inter- and intramolecular interactions of $\alpha$-lactalbumin XI. Comparison of the "exposure" of tyrosyl, tryptophyl, and lysyl side chains in the goat and bovine proteins. Biochim. Biophys. Acta 285:124-144.

Liu, Y., and R. Guo. 2008a. Aggregation properties of aqueous casein hydrolysate solutions at different pH. Int. Dairy J. 18:1022-1027.
Liu, Y., and R. Guo. 2008b. pH-dependent structures and properties of casein micelles. Biophys. Chem. 136:67-73.

McMahon, D. J., H. Du, W. R. McManus, and K. M. Larsen. 2009. Microstructural changes in casein supramolecules during acidification of skim milk. J. Dairy Sci. 92:5854-5867.

Nakamura, A., H. Furuta, M. Kato, H. Maeda, and Y. Nagamatsu. 2003. Effect of soybean soluble polysaccharides on the stability of milk protein under acidic conditions. Food Hydrocoll. 17:333-343.

Nakamura, A., H. Furuta, H. Maeda, Y. Nagamatsu, and A. Yoshimoto. 2001. Analysis of structural components and molecular construction of soybean soluble polysaccharides by stepwise enzymatic degradation. Biosci. Biotechnol. Biochem. 65:2249-2258.

Nakamura, A., R. Yoshida, H. Maeda, and M. Corredig. 2006. The stabilizing behaviour of soybean soluble polysaccharide and pectin in acidified milk beverages. Int. Dairy J. 16:361-369.

Nobuhara, T., K. Matsumiya, Y. Nambu, A. Nakamura, N. Fujii, and Y. Matsumura. 2014. Stabilization of milk protein dispersion by soybean soluble polysaccharide under acidic $\mathrm{pH}$ conditions. Food Hydrocoll. 34:39-45.

Pereyra, R. K. A. Schmidt, and L. Wicker. 1997. Interaction and stabilization of acidified casein dispersions with low and high methoxyl pectins. J. Agric. Food Chem. 45:3448-3451.

Rahimi, Y. S., and M. Corredig. 2012. Heating of milk alters the binding of curcumin to casein micelles. A fluorescence spectroscopy study. Food Chem. 132:1143-1149.

Sahoo, D., V. Narayanaswami, C. M. Kay, and R. O. Ryan. 2000. Pyrene excimer fluorescence: A spatially sensitive probe to monitor lipid-induced helical rearrangement of apolipophorin III. Biochemistry 39:6594-6601.

Tayeh, N., T. Rungassamy, and J. R. Albani. 2009. Fluorescence spectral resolution of tryptophan residues in bovine and human serum albumins. J. Pharm. Biomed. Anal. 50:107-116.

Vanhooren, A., E. Illyes, Z. Majer, and I. Hanssens. 2006. Fluorescence contributions of the individual Trp residues in goat $\alpha$-lactalbumin. Biochim. Biophys. Acta 1764:1586-1591. 